\title{
Effects of Melatonin for Delirium in Elderly Acute Heart Failure Patients: A Randomized, Single-Center, Double-Blind, and Placebo-Controlled Trial
}

\author{
Bi Yin, BS, Ting Ye, MM, Yinxia Liu, BS, Rong Wan, BS, Lilei Gu, BS, Gangjun Zong, MD \\ Department of Cardiology, Wuxi Clinical Medical School of Anhui Medical University, 904th Hospital of PLA, Wuxi, China
}

\section{ABSTRACT}

Background: Delirium is a common, life-threatening, typical clinical syndrome with the main clinical manifestations of temporary organic mental disorder without specific drug treatment. The aim of the study was to explore the benefits of melatonin for the treatment of delirium after acute heart failure in elderly patients.

Methods: This was a randomized, double-blind, and placebo-controlled trial. This study enrolled patients aged more than 60 years after acute heart failure. A computer-generated randomization sequence (in a 1:1 ratio) was used to randomly assign patients to receive either melatonin ( $3 \mathrm{mg} /$ day, 7 days) or placebos. The primary endpoint was the incidence of delirium, assessed twice daily with the Confusion Assessment Method during the first 7 days. Analyses were performed by intention-to-treat and safety populations.

Results: Between October 2015 and October 2019, 584 patients were assessed. A total of 497 patients randomly were assigned to receive either placebo $(N=249)$ or melatonin $(N=$ 248). The incidence of delirium was significantly lower in the melatonin group than in the placebo group $(27.0 \%$ vs. $36.9 \%$, $P=0.021)$. Regarding safety, the occurrence of rhabdomyolysis and abnormal hepatic function did not differ between the two groups.

Conclusion: The current study (clinical trial registered number: CHWX-904-201511) suggests that acute melatonin treatment can reduce the incidence of delirium for elderly acute heart failure. It also can reduce the time of hospital stays and hospitalization costs. The therapy was safe and worth spreading.

\section{INTRODUCTION}

Delirium is a common, life-threatening, typical clinical syndrome with the main clinical manifestations of temporary organic mental disorder, acute brain dysfunction, changes in

Received October 1, 2021; accepted November 3, 2021.

Correspondence: Lilei Gu, Wuxi Clinical Medical School of Anbui Medical University, Department of Cardiology, 904th Hospital of PLA, Xing Yuan North Road 101, Wuxi, 214044, China, Telephone +86 051085142441, Fax +86051085142431 (e-mail:176233764@qq.com). cognitive function and disorientation that can lead to longterm cognitive impairment and increased disability, mortality, length of hospital stay, and hospitalization costs [Gleason 2015]. More than $20 \%$ of patients who stay in the intensive care unit (ICU) are diagnosed with delirium [Mather 2017]. In recent years, with the growth in the aging population, the number of older patients undergoing surgery has increased, and the incidence of postoperative delirium after surgery in older patients has been reported to be as high as $46 \%$ [Saczynski 2012]. In the past 20 years, the treatment of delirium in older patients has become a severe challenge. As the elderly population grows, an increasing number of patients suffer from acute myocardial infarction, coronary artery sclerosis, and acute heart failure [Global, Regional, and National Burden of Stroke 2019]. The incidence of delirium in elderly patients is increasing. Pak et al. [Pak 2020] also reported that the incidence of delirium was $27.3 \%$ in older adult patients with acute decompensated heart failure, associated with a higher 90-day all-cause mortality. However, the diagnosis and treatment of delirium are relatively difficult, and there is no specific drug treatment, which also makes the treatment of delirium severely challenging. To date, most drugs currently available in the clinic have no specific effect on delirium, and there also is a lack of evidence-based medicine for the treatment of the disease.

Melatonin (N-acetyl-5-methoxytryptamine; Mel), secreted by the pineal gland, is a potent free-radical scavenger and broad-spectrum antioxidant that easily passes through the blood brain barrier (BBB) without toxicity in large doses [Chen 2014]. Han et al. [Han 2020] reported a retrospective study in which Mel and its analogs were effective in the treatment of delirium, but more evidence is needed to confirm their efficacy after acute heart failure (AHF). In particular, the efficacy of Mel in the treatment of delirium in patients after AHF remains unclear. The present study, therefore, explored whether Mel treatment could reduce delirium and improve clinical outcomes after acute heart failure.

\section{METHODS}

Study design: This was a randomized, double-blind, and parallel arm placebo-controlled trial in Jiangsu between October 1, 2015, and October 1, 2019. The study was designed to assess the superiority of intervention. The study protocol was approved by the Anhui Medical University Affiliated Wuxi Clinical College Clinical Research Ethics Committees 
(2015-YXLL-021). The study protocol received Ethics Committee approval from all participating centers. Written informed consent was obtained from patients whose competence was established by their accurate orientation for time, place, and person, as well as an understanding of the recruiter's description of the trial or otherwise from their next of kin or their legal representative. Patients randomly were assigned (1:1) to receive $3 \mathrm{mg} /$ day of Mel or placebos within 7 days after AHF. (Figure 1) Mel or placebos orally were administered for up to 7 days after AHF. The final follow up was 30 days after AHF.

Study patients, inclusion criteria, and exclusion criteria: Eligible patients were more than 60 years old with $\mathrm{AHF}$ in the ICU. Inclusion criteria were as follows: (1) Age more than 60 years; (2) Could be randomized and received Mel or placebo within 7 days after surgery, and (3) Acute AHF patients were admitted to the ICU. Exclusion criteria were as follows: (1) Unsalvageable patients likely on admission; (2) High cholesterol combined with diabetes; (3) Combined with brain injury or neurosurgery history; (4) Neurologic disease; (5) Patients with a history of mental illness and epilepsy; and (6) Researchers found other reasons.

Randomization and masking: Permuted-block randomization was performed using a computer system with an allocation list generating random numbers (in a 1:1 ratio), using SPSS 14.0 software (SPSS Institute, Hefei, Anhui Medical University). This was performed by a statistician not associated with the project team to protect the blinding and integrity of the study. Results of randomization were sealed in sequentially numbered envelopes and stored at the site of the investigation until the end of the study. During the study period, all included patients randomly were assigned to receive $3 \mathrm{mg}$ /day of Mel or placebos within 7 days after acute AHF (Figure 1). A study nurse administered the study drugs, according to the randomization sequence. Both the study members and patients were blinded to the study drug allocation. If an emergency occurred, like a severe hepatic failure, then two experts could request unmasking of the treatment allocation or adjust or interrupt study drug, if necessary. All situations were documented. Patient demographics, medical histories, and relevant investigation results were collected.

Outcome assessment and the primary endpoint: All clinical and imaging data were assessed by a masked independent diagnostic and assessment committee. This committee included two researchers who were trained before the study and were not involved in the clinical care of patients. The primary endpoint was an incidence of delirium in the first 7 days after acute AHF. The first delirium was evaluated around 24 hours after acute AHF and evaluated twice daily (from 6 to 8 am and from 6 to $8 \mathrm{pm}$ ). Delirium was the assessment by Confusion Assessment Method (CAM) and the CAM for the Intensive Care Unit (CAM-ICU). Both CAM and CAM-ICU detected 4 features of delirium: (1) Acute onset of mental status changes or a fluctuating course; (2) Inattention; (3) Disorganized thinking; and (4) Altered level of consciousness. To be diagnosed as delirious, a patient displayed features 1 and 2, with either 3 or 4 .

The second endpoints: The second endpoints included all-cause 30-day mortality, length of stay in the ICU, and occurrence of non-delirium complications and hospital costs.

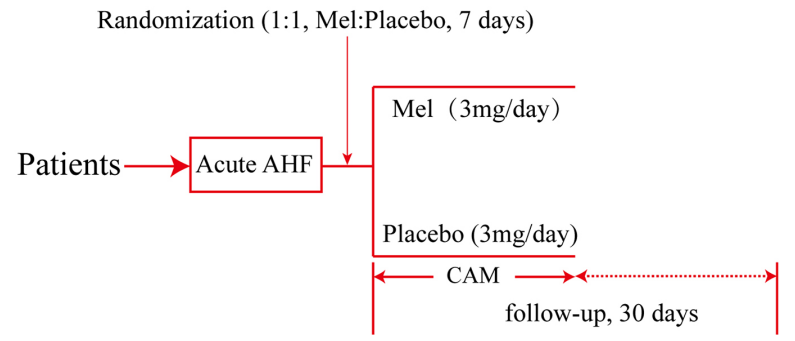

Figure 1. Study design

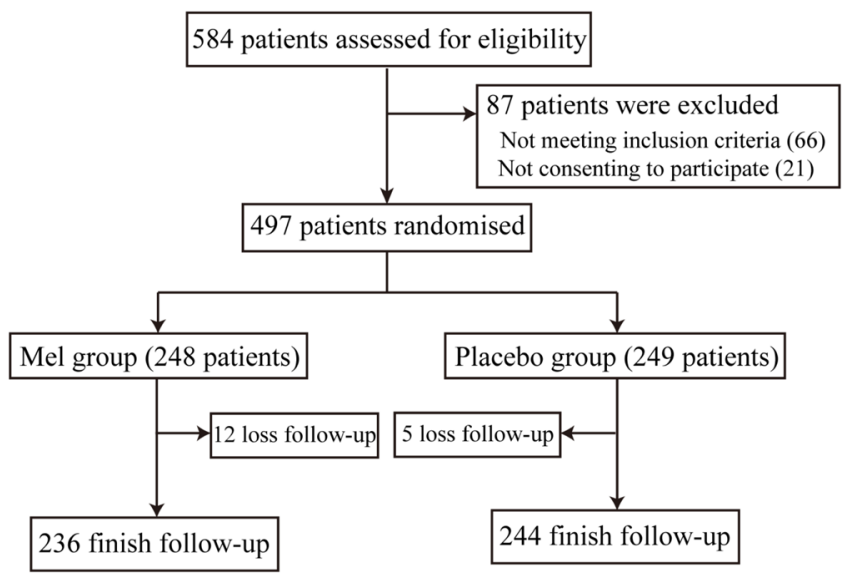

Figure 2. Trial profile

Statistical analysis: Based on previous data, it was estimated that 400 patients would be required to confirm these effects with an $\alpha$ of $5 \%$ and $80 \%$ power. Further assuming a $10 \%$ loss to follow up, 497 patients were enrolled [Hokuto 2020]. A research nurse entered all baseline and outcome data in the study database. Data were collected on handwritten forms and archived in a password-protected, electronic database. All continuous variables are presented as the mean \pm standard deviation. SPSS 14.0 statistical software (SPSS, Inc., Chicago, Illinois, USA) was used for the statistical analyses. Independent two-sample t-tests and Spearman correlations were used to assess categorical data. Fisher's exact t-test was used to compare categorical data between the two groups, and the Mann-Whitney U test was used to compare ordinal or continuous variables between groups. A value of $P<0.05$ was considered statistically significant.

\section{RESULTS}

Between October 1, 2015, and October 1, 2019, 584 patients were assessed. A total of 497 randomly were assigned to receive either placebo $(N=249)$ or $\mathrm{Mel}(N=248)$. (Figure 2) During the study period, there were no opening-blind cases, and there was no statistically significant difference in baseline data between the two groups. (Table 1) During the study period, 17 patients lost 30 days of follow up (5 in the control group and 12 in the Mel group). All other patients 
Table 1. Demographic and baseline characteristics of the study population in the two groups

\begin{tabular}{lccc}
\hline & Placebo & Mel & $P$-value \\
\hline Number of patients & 249 & 248 & \\
Age (mean \pm SD) & $68.5 \pm 7.1$ & $69.1 \pm 7.5$ & 0.36 \\
Gender & - & - & 0.38 \\
Male & 143 & 152 & \\
Female & 106 & 96 & \\
History of hypertension & - & - & 0.28 \\
Yes & 195 & 184 & \\
No & 54 & 64 & \\
Nicotine use & - & - & 0.71 \\
Yes & 72 & 68 & \\
No & 177 & 180 & \\
& & & \\
& 50 &
\end{tabular}

Table 2. Comparison of Primary endpoint-clinical outcomes between the two groups

\begin{tabular}{lccc}
\hline & Placebo & Mel & P-value \\
\hline Number of patients & 249 & 248 & \\
Delirium & $36.9 \%$ & $27 \%$ & 0.02 \\
& $(92 / 249)$ & $(67 / 248)$ & \\
30-day all-cause mortality & $44.7 \%$ & $38.1 \%$ & 0.15
\end{tabular}

Table 3. Comparison of Secondary endpoint between the two groups

\begin{tabular}{lccc}
\hline & Placebo & Mel & P-value \\
\hline $\begin{array}{l}\text { Number of patients } \\
\text { Hospital stay } \\
\text { (day, mean } \pm \text { SD) }\end{array}$ & 249 & 248 & \\
$\begin{array}{l}\text { Hospitalization costs } \\
(\$ 1000, \text { mean } \pm \text { SD) }\end{array}$ & $5.9 \pm 2.6$ & $6.5 \pm 2.2$ & 0.01
\end{tabular}

were included in the final intention-to-treat analyses (Figure 2). The final visit of the last randomized patient was done on December 22, 2019.

Primary endpoint-clinical outcomes: The overall incidence of delirium after acute AHF in elderly patients was $32.0 \%(159 / 497)$. The incidence of delirium was $27 \%$ $(67 / 248)$ in the Mel group and 36.9\% (92/249) in the placebo control group at 7 days after acute AHF. The incidence of delirium was significantly higher in the placebo group than in the Mel group, and it was statistically significant $(P=0.021)$. After 30 days of follow up, 17 patients were not included in the mortality rate as lost follow up after discharge. The 30-day all-cause mortality rate of the Mel group was 38.1\% $(90 / 236)$, while that of the placebo control group was $44.7 \%$ $(109 / 244)$, with no significant statistical difference $(P=$ 0.146). (Table 2)

Secondary endpoint: Previous clinical studies found that hospital stay and hospitalization costs of patients with delirium significantly increased, so the present study also compared the average length of hospital stay and hospitalization costs between the two groups. The mean length of stay in the Mel group was 18.1 days, and the mean length of stay in the placebo group was 19.8 days, with a statistically significant difference between the two groups $(P=0.01)$. The average hospitalization cost of the Mel group was $\$ 5850$, which was less than $\$ 6470$ in the placebo control group. This was statistically significant $(P=0.01)$. (Table 3$)$

Safety evaluation-complications: Two cases occurred with mild diarrhea and vomiting and may be related to the Mel. They got better with little or no treatment. Sixteen mild liver dysfunction cases were reported in the control group, while there were 25 cases of mild liver dysfunction in the Mel group. These improved after liver protection treatment, and it was significantly different between the two groups. No blind cases were opened during the treatment period, and 17 patients were lost to follow up after discharge and did not participate in the mortality statistics, and no shedding cases were found.

\section{DISCUSSION}

The present study found that in elderly patients after acute AHF, prophylactic low-dose Mel orally after acute AHF can significantly decrease the incidence of delirium. Mel supplementation improved sleep after surgery. It also shortened the length of stay in the hospital and decreased hospitalization expenses, while complications, such as liver dysfunction, were similar. Also, low-dose Mel improved the 30-day all-cause mortality rate.

The overall incidence of delirium in older patients, following acute $\mathrm{AHF}$, was $32.0 \%$, similar to previous reports [Pak 2020]. Pak et al. [Pak 2020] also evaluated by multivariable Cox and logistic regression analyses, found that deliriumrelated risk factors included advanced age and male sex, and that delirium can increase the mortality in older patients. In the present study, we also found that the 30-day all-cause mortality rate of the Mel group was $38.1 \%$, which is higher than the control group, and no statistical significance may be related to the sample size. Kwak et al. [Kwak 2021] conducted a retrospective observational study from 2011 to 2014 and enrolled 568,565 patients 65 years or older with acute heart failure; they found the overall prevalence of delirium was $4.53 \%$, and delirium can increase the total hospital cost and the length of stay. In the present study, decreased delirium can help lower hospital costs and length of stay. Iwata et al. [Iwata 2020] reported that the in-hospital mortality rate was significantly higher in patients with delirium, and nursing home residential status and dementia independently were associated with the development of delirium. 
Melatonin is a free radical scavenger by the pineal gland [Liu 2019] that cannot only improve vasospasm and early brain damage, but also significantly improve sleep disorders and reduce the incidence of delirium [Han 2020]. Unexpectedly, the anti-delirium role of Mel has always been a controversial issue. Jaiswal [SJ 2019] demonstrated that $3 \mathrm{mg} \mathrm{Mel}$ did not reduce the incidence of delirium after endovascular treatment with pulmonary thrombocytes in a randomized controlled trial, and the same study team also demonstrated that Mel did not improve delirium or sleep in patients with severe hospitalization in a prospective randomized controlled study [SJ 2018]. However, Yang [CP 2020] also found that low doses of Mel can decrease the incidence of delirium through a meta-analysis. In the basic research, Mel can improve the mitochondrial dysfunction associated with heart failure caused by isoprenaline hydrochloride [Odinokova 2018]. Additionally, previous study demonstrated that pretreatment with Mel can alleviate renal ischemia-reperfusion injury-mediated cardiac damage by regulating myocardial diastolic function and decreasing myocardial cell injury, thereby alleviating cytoplasmic and mitochondrial calcium overload [Wang 2020]. However, there was no evidence regarding the effect of $\mathrm{Mel}$ on delirium in elderly patients with acute AHF.

In the present study, Mel significantly improved the incidence of delirium in elderly patients after acute AHF, but it did not improve the 30-day all-cause mortality after acute AHF. The specific molecular mechanism of the anti-delirium effects after Mel administration in elderly delirium after acute AHF may be closely related to its anti-vasospasm effects, antineuronal apoptosis, and improvements in cerebral perfusion and sleep quality [Chen 2020]. The study also found that patients' length of stay in the intensive care unit and hospitalization costs were significantly reduced as the incidence of delirium decreased.

The limitations of this study were as follows:

1. We collected key baseline and outcome data but did not call patients back for study visits for detailed assessments of the quality of life or activities of daily living (ADL).

2. All participants were screened and enrolled after ICU admission and did not have baseline delirium assessments with cognitive function assessments.

3. This study used a single dose of Mel. The conventional low dose may not work well.

4. This study did not take into account clinical factors, such as subjective sleep quality, pain, the daily prevalence of delirium, and time of endotracheal intubation.

\section{CONCLUSION}

The results from this trial demonstrated that Mel therapy reduces delirium after acute AHF. No evidence was found that low-dose Mel increased complications. Additionally, it significantly decreased hospitalization expenses and hospitalization time. Whether the favorable effects afforded by this novel application of Mel will result in improved long-term outcomes remains unknown. Further investigation of patients undergoing acute $\mathrm{AHF}$ and the effects of different doses are necessary to fully understand the potential usefulness of Mel in elderly patients.

\section{ACKNOWLEDGEMENT}

We would like to acknowledge the technical and language support of Jiangsu Brilliant Biological Technology Co., Ltd. Funding: Top Talent Support Program for Young and Middle-Aged People of Wuxi Health Committee.

\section{REFERENCES}

Chen J, Chen G, Li J, et al. 2014. Melatonin attenuates inflammatory response-induced brain edema in early brain injury following a subarachnoid hemorrhage: a possible role for the regulation of pro-inflammatory cytokines. Journal of Pineal Research. Oct;57(3):340-347.

Chen J, Wang Y, Hu X, et al. 2020. The role of statins in the management of delirium: Recent advances. CNS Neurol Disord Drug Targets. Jul 19.

CP Y, PT T, J P-CC, H S, SK S, KP S. 2020. Melatonergic agents in the prevention of delirium: A network meta-analysis of randomized controlled trials. Sleep medicine reviews. 50:101235.

Gleason LJ, Schmitt EM, Kosar CM, et al. 2015. Effect of Delirium and Other Major Complications on Outcomes After Elective Surgery in Older Adults. Jama Surgery. Dec; 150(12):1134-1140.

Global, regional, and national burden of stroke, 1990-2016: a systematic analysis for the Global Burden of Disease Study 2016. 2019. Lancet Neurol. May;18(5):439-458.

Han YY, Wu J, Qin ZS, et al. 2020. Melatonin and its analogues for the prevention of postoperative delirium: A systematic review and metaanalysis. Journal of Pineal Research. e12644.

Hokuto D, Nomi T, Yoshikawa T, Matsuo Y, Kamitani N, Sho M. 2020. Preventative effects of ramelteon against postoperative delirium after elective liver resection. PLoS One. 15(11):e0241673.

Iwata E, Kondo T, Kato T, et al. 2020. Prognostic Value of Delirium in Patients With Acute Heart Failure in the Intensive Care Unit. Can J Cardiol. Oct;36(10):1649-1657.

Kwak MJ, Avritscher E, Holmes HM, et al. 2021. Delirium Among Hospitalized Older Adults With Acute Heart Failure Exacerbation. J Card Fail. Apr;27(4):453-459.

Liu J-C, Li L, Yan H-C, et al. 2019. Identification of oxidative stressrelated Xdh gene as a di(2-ethylhexyl)phthalate (DEHP) target and the use of melatonin to alleviate the DEHP-induced impairments in newborn mouse ovaries. Journal of Pineal Research. Aug;67(1)e12577.

Mather JF, Corradi JP, Waszynski C, et al. 2017. Statin and Its Association With Delirium in the Medical ICU. Critical Care Medicine. Sep;45(9):1515-1522.

Odinokova I, Baburina Y, Kruglov A, et al. 2018. Effect of Melatonin on Rat Heart Mitochondria in Acute Heart Failure in Aged Rats. Int J Mol Sci. May 23; 19(6).

Pak M, Hara M, Miura S, et al. 2020. Delirium is associated with high mortality in older adult patients with acute decompensated heart failure. BMC Geriatr. Dec 3;20(1):524.

Saczynski JS, Marcantonio ER, Quach L, et al. 2012. Cognitive 
trajectories after postoperative delirium. N Engl J Med. Jul 5;367(1):30-9.

SJ J, AD V, AJ H, et al. 2019. Ramelteon for Prevention of Postoperative Delirium: A Randomized Controlled Trial in Patients Undergoing Elective Pulmonary Thromboendarterectomy. Critical care medicine. 47(12):1751-1758.

SJ J, TJ M, NE W, et al. 2018. Melatonin and Sleep in Preventing
Hospitalized Delirium: A Randomized Clinical Trial. The American journal of medicine. 131(9):1110-1117.e4.

Wang J, Toan S, Li R, Zhou H. 2020. Melatonin fine-tunes intracellular calcium signals and eliminates myocardial damage through the IP3R/ MCU pathways in cardiorenal syndrome type 3. Biochem Pharmacol. Apr;174:113832. 